\title{
Synthetic Chemistry
}

\section{4,4'-Diiododiphenylmethane}

To a solution of 4,4'-diaminodiphenylmethane (5.0 g, $25 \mathrm{mmol})$ in conc. sulphuric acid $(20 \mathrm{ml})$ at $0^{\circ} \mathrm{C}$ was added dropwise a solution of sodium nitrite $(5.0 \mathrm{~g}, 72 \mathrm{mmol})$ in water $(10 \mathrm{ml})$ over a period of 10 minutes. The resulting dark red mixture was stirred at $0^{\circ} \mathrm{C}$ for a further 30 minutes before a solution of potassium iodide $(29.0 \mathrm{~g}, 170 \mathrm{mmol})$ in water $(100 \mathrm{ml})$ was added. The mixture was heated to $50^{\circ} \mathrm{C}$ for $1 \mathrm{~h}$. then cooled to room temperature and neutralised with $50 \%$ aqueous sodium hydroxide. The mixture was extracted with DCM and the combined organics washed with $1 \mathrm{M} \mathrm{HCl}$ and dried over $\mathrm{MgSO}_{4}$. Concentration in vacuo yielded a dark red oil which was purified by flash chromatography on silica gel eluting with 40-60 petrol to yield $4 \mathrm{c}(5.7 \mathrm{~g}, 54 \%)$ as a white solid; m.p. 91-93 ${ }^{\circ} \mathrm{C}$ (lit., $\left.{ }^{1} 85-86^{\circ} \mathrm{C}\right) ; \delta_{H}\left(200 \mathrm{MHz}, \mathrm{CDCl}_{3}\right) 3.87\left(2 \mathrm{H}, \mathrm{s}, \mathrm{Ar}_{2} \mathrm{CH}_{2}\right), 6.49$ $\left(4 \mathrm{H}, \mathrm{d}, J=8.3 \mathrm{~Hz}, 2-, 2^{\prime}-, 6^{-}, 6{ }^{\prime}-H\right), 7.62\left(4 \mathrm{H}, \mathrm{d}, J=8.3 \mathrm{~Hz}, 3-, 3^{\prime}-\right.$, 5-, 5'-H) ppm; $\delta_{C}$ $\left(100 \mathrm{MHz}, \mathrm{DMSO}-\mathrm{d}_{6}\right) 40.9\left(\mathrm{Ar}_{2} \mathrm{CH}_{2}\right), 91.6$ (4-, 4'-C), 130.9 (2-, 2'-, 6-, 6'-C), 137.6 (3-, 3'-, 5-, 5'-C), 140.1 (1-, 1'-C) ppm; $v_{\max }\left(\mathrm{CH}_{2} \mathrm{Cl}_{2}\right.$ thin film) 1482, 1435, 1397, 1109, $1007,857,806,774 \mathrm{~cm}^{-1} ; \mathrm{m} / z\left(\mathrm{EI}^{+}\right) 420\left(\mathrm{M}^{+}, 100 \%\right)$.

\section{4,4'-Diiodobenzophenone}

To a suspension of 4,4'-Diiododiphenylmethane $(2.75 \mathrm{~g}, 6.5 \mathrm{mmol})$ in a mixture of glacial acetic acid $(25 \mathrm{ml})$ and water $(10 \mathrm{ml})$ was added chromium oxide $(3.27 \mathrm{~g}, 33.0$ mmol). The mixture was heated to reflux for $24 \mathrm{~h}$. then thrown into ice water $(250 \mathrm{ml})$. The mixture was filtered and the resulting solid washed with water then recrystallised from toluene to yield $\mathbf{5}(2.1 \mathrm{~g}, 70 \%)$ as a white crystalline solid; m.p. $233-235^{\circ} \mathrm{C}$ (lit., ${ }^{2}$ $\left.234^{\circ} \mathrm{C}\right) ; \delta_{H}\left(200 \mathrm{MHz}, \mathrm{CDCl}_{3}\right) 7.50\left(4 \mathrm{H}, \mathrm{d}, J=8.5 \mathrm{~Hz}, 2-, 2^{\prime}-, 6^{-}, 6\right.$ 6'-H), $7.87(4 \mathrm{H}, \mathrm{d}, J=$ $8.5 \mathrm{~Hz}, 3-, 3^{\prime}-$, 5-, 5'-H) ppm; $\delta_{C}\left(100 \mathrm{MHz}, \mathrm{CDCl}_{3}\right) 100.5$ (4-, 4'-C), 131.3 (2-, 2'-, 6-, 6'-C), 136.4 (1-, 1'-C), 137.7 (3-, 3'-, 5-, 5'-C), $195.1\left(\mathrm{Ar}_{2} \mathrm{C}=\mathrm{O}\right) \mathrm{ppm} ; v_{\max }\left(\mathrm{CH}_{2} \mathrm{Cl}_{2}\right.$ thin film) $1644,1391,1279,851,820,743 \mathrm{~cm}^{-1} ; \mathrm{m} / z\left(\mathrm{EI}^{+}\right) 434\left(\mathrm{M}^{+}, 100 \%\right)$.

\section{4,4'-Diodobenzophenone hydrazone}

To a solution of 4,4'-Diiodobenzophenone $(1.50 \mathrm{~g}, 3.5 \mathrm{mmol})$ in ethanol $(50 \mathrm{ml})$ was added hydrazine hydrate $(1.70 \mathrm{~g}, 35.0 \mathrm{mmol})$. The mixture was heated to reflux for $18 \mathrm{~h}$. then concentred in vacuo. The residue was partioned between DCM and water and the organic layer collected and dried over $\mathrm{MgSO}_{4}$. Concentration in vacuo yielded a inseparable mixture of azine impurity and cis/trans $6 \mathbf{6}(1.52 \mathrm{~g}, 97 \%)$ as a yellow oil; $\delta_{H}$ $\left(200 \mathrm{MHz}, \mathrm{CDCl}_{3}\right) 5.48\left(2 \mathrm{H}, \mathrm{bs}, \mathrm{NNH}_{2}\right), 7.03(2 \mathrm{H}, \mathrm{d}, J=8.6 \mathrm{~Hz}, 2-, 6-H), 7.17(2 \mathrm{H}, \mathrm{d}, J$ $\left.=8.8 \mathrm{~Hz}, 2^{\prime}-, 6^{\prime}-H\right) 7.62\left(2 \mathrm{H}, \mathrm{d}, J=8.8 \mathrm{~Hz}, 3^{\prime}-, 5^{\prime}-H\right), 7.88(2 \mathrm{H}, \mathrm{d}, J=8.6 \mathrm{~Hz}, 3-, 5-H)$ ppm; $\delta_{C}\left(100 \mathrm{MHz}, \mathrm{CDCl}_{3}\right)$ 94.1, 95.2 (4-, 4'-C), 128.0, 130.6 (2-, 2'-, 6-, 6'-C), 137.1,137.3 (1-, 1'-C), 137.2, 138.7 (3-, 3'-, 5-, 5'-C), $146.5\left(C=\mathrm{NNH}_{2}\right) \mathrm{ppm} ; v_{\max }$ $\left(\mathrm{CH}_{2} \mathrm{Cl}_{2}\right.$ thin film) $3407,3287,3047,2985,1589,1483,1390,1328,1060,1006,824 \mathrm{~cm}^{-}$ 1 


\section{Bis(4-iodophenyl) diazomethane}

To a mixture of yellow mercury (II) oxide $(0.08 \mathrm{~g}, 0.040 \mathrm{mmol})$, sodium sulphate $(0.06 \mathrm{~g}$, $0.047 \mathrm{mmol})$ and saturated $\mathrm{KOH}$ in $\mathrm{EtOH}(1 \mathrm{ml})$ was added a solution of 4,4'Diodobenzophenone hydrazone $(0.15 \mathrm{~g}, 0.034 \mathrm{mmol})$ in DCM $(10 \mathrm{ml})$. The mixture was stirred in the dark for $5 \mathrm{~h}$. then filtered through Celite. The filtrate was collected and concentrated and the residue purified by flash chromatography on silica gel $(9: 1$ Petrol:diethyl ether) to furnish the product $(0.14 \mathrm{~g}, 93 \%)$ a dark purple solid; m.p. 98$101^{\circ} \mathrm{C} ; \delta_{H}\left(200 \mathrm{MHz}, \mathrm{DMSO}-\mathrm{d}_{6}\right) 7.08\left(4 \mathrm{H}, \mathrm{d}, J=8.6 \mathrm{~Hz}, 2-, 2^{\prime}-, 6^{-}, 6\right.$ ' $\left.-H\right), 7.76(4 \mathrm{H}, \mathrm{d}, J$ $=8.6 \mathrm{~Hz}, 3-, 3^{\prime}-$, 5-, 5'-H) ppm; $\delta_{C}\left(100 \mathrm{MHz}, \mathrm{DMSO}-\mathrm{d}_{6}\right) 90.8$ (4-, 4'-C), 126.9 (2-, 2'-, 6-, 6'-C), $128.1\left(1-, 1^{\prime}-C\right), 138.0\left(3-, 3^{\prime}-\right.$, 5-, 5'-C) ppm; $v_{\max }\left(\mathrm{CH}_{2} \mathrm{Cl}_{2}\right.$ thin film) $2035 \mathrm{~cm}^{-}$

\section{4,4'-Dinitrodiphenylmethane}

To a mixture of concentrated sulphuric acid $(17 \mathrm{ml})$ and concentrated nitric acid $(19 \mathrm{ml})$ at $-40^{\circ} \mathrm{C}$ was added diphenylmethane $(5.09 \mathrm{~g}, 30 \mathrm{mmol})$. The mixture was stirred at $-40^{\circ} \mathrm{C}$ for $5 \mathrm{~h}$ then thrown into ice water $(100 \mathrm{ml})$. The resulting solid was collected by filtration and washed with water. Recrystallisation from toluene yielded the product $(4.27 \mathrm{~g}, 63 \%)$ a white solid; m.p. $181-185^{\circ} \mathrm{C}$ (toluene) (lit., $\left.{ }^{3} 187.4-188.4^{\circ} \mathrm{C}\right) ; \delta_{H}\left(200 \mathrm{MHz}, \mathrm{CDCl}_{3}\right) 4.20$ $\left(2 \mathrm{H}, \mathrm{s}, \mathrm{Ar}_{2} \mathrm{CH}_{2}\right), 7.46\left(4 \mathrm{H}, \mathrm{d}, J=9.1 \mathrm{~Hz}, 3-, 3^{\prime}-, 5_{-}, 5\right.$ ' $\left.-H\right), 8.20\left(4 \mathrm{H}, \mathrm{d}, J=9.1 \mathrm{~Hz}, 2-, 2^{\prime}-\right.$ , 6-, 6'-H) ppm; $\delta_{C}\left(100 \mathrm{MHz}, \mathrm{DMSO}-\mathrm{d}_{6}\right) 40.1\left(\mathrm{Ar}_{2} \mathrm{CH}_{2}\right), 123.8$ (3-, 3'-, 5-, 5'-C), 130.2 (2-, 2'-, 6-, 6'-C), 146.2 (4-, 4'-C), 148.0 (1-, 1'-C) ppm; $v_{\max }\left(\mathrm{CH}_{2} \mathrm{Cl}_{2}\right.$ thin film) 1601, $1509,1342,1108,884 \mathrm{~cm}^{-1} ; \mathrm{m} / z\left(\mathrm{ES}^{-}\right) 257\left([\mathrm{M}-\mathrm{H}]^{-}, 100 \%\right)$.

\section{4,4'-Dinitrobenzophenone}

To a suspension of 4,4'-Dinitrodiphenylmethane (4.27 g, $19 \mathrm{mmol})$ in acetic acid (64 ml) was added a solution of chromium trioxide $(8.18 \mathrm{~g}, 80 \mathrm{mmol})$ in water $(42 \mathrm{ml})$. the mixture was heated to reflux for $24 \mathrm{~h}$, then thrown into ice water $(100 \mathrm{ml})$. The resulting solid was filtered, washed with water and aqueous sat. sodium hydrogen carbonate. The solid was recrystallised from toluene to yield the product $(4.21 \mathrm{~g}, 93 \%)$ as pale yellow crystals; m.p. $185-187^{\circ} \mathrm{C}$ (toluene) (lit., $\left.{ }^{4} 188-191.5^{\circ} \mathrm{C}\right) ; \delta_{H}\left(400 \mathrm{MHz}, \mathrm{CDCl}_{3}\right) 7.97(4 \mathrm{H}, \mathrm{d}$, $J=9.0 \mathrm{~Hz}, 2-, 2^{\prime}-$, 6-, 6'- $H$ ), 8.39 (4H, d, $J=9.0 \mathrm{~Hz}, 3-, 3^{\prime}-$, 5-, 5'-H) ppm; $\delta_{C}(100 \mathrm{MHz}$, $\left.\mathrm{CDCl}_{3}\right) 123.4$ (3-, 3'-, 5-, 5'-C), 130.8 (2-, 2'-, 6-, 6'-C), 141.2 (1-, 1'-C), 150.3 (4-, 4'C), $193.0(\mathrm{C}=\mathrm{O}) \mathrm{ppm} ; v_{\max }\left(\mathrm{CH}_{2} \mathrm{Cl}_{2}\right.$ thin film) 1671, 1602, 1520, 1351, 1271, 932, 847

$\mathrm{cm}^{-1} ; \mathrm{m} / \mathrm{z}\left(\mathrm{TOF} \mathrm{EI} \mathrm{I}^{+}\right) 272\left(\mathrm{M}^{+}, 100 \%\right)$; found $\left(\mathrm{TOF} \mathrm{EI}^{+}\right) 272.0430, \mathrm{C}_{13} \mathrm{H}_{8} \mathrm{~N}_{2} \mathrm{O}_{5}$ requires 272.0433 .

\section{4,4'-Dinitrobenzophenone (4'’-toluene sulphonyl) hydrazone}

To a solution of 4,4'-dintrobenzophenone (1.85 $\mathrm{g}, 68 \mathrm{mmol})$ in a mixture of ethanol (10 $\mathrm{ml})$ and THF $(10 \mathrm{ml})$ was added $p$-toulenesulfonyl hydrazide $(1.90 \mathrm{~g}, 100 \mathrm{mmol})$. The mixture was heated to reflux for $24 \mathrm{~h}$ then concentrated in vacuo. The residue was washed with ethanol then recrystallised from toluene to yield the product $(2.6 \mathrm{~g}, 87 \%)$ as 
pale yellow crystals; m.p. $175-178^{\circ} \mathrm{C}$ (toluene) (lit., $\left.{ }^{5} 179-180^{\circ} \mathrm{C}\right) ; \delta_{H}(400 \mathrm{MHz}, \mathrm{DMSO}-$ d $\left.{ }_{6}\right) 2.40\left(3 \mathrm{H}, \mathrm{s}, \mathrm{ArCH}_{3}\right), 7.45(2 \mathrm{H}, \mathrm{d}, J=8.3 \mathrm{~Hz}, 2$ ' '-, 6' '-H), $7.53(2 \mathrm{H}, \mathrm{d}, J=8.9 \mathrm{~Hz}, 2-$, 6- $H$ or 2'-, 6'-H), $7.58(2 \mathrm{H}, \mathrm{d}, J=8.8 \mathrm{~Hz}, 2-, 6-H$ or 2'-, 6'- $H), 7.81(2 \mathrm{H}, \mathrm{d}, J=8.3 \mathrm{~Hz}$, 3' '-, 5' '-H), ), $8.21(2 \mathrm{H}, \mathrm{d}, J=8.9 \mathrm{~Hz}, 3-, 5-H$ or 3'-, 5'-H), 8.39 (2H, d, $J=8.8 \mathrm{~Hz}, 3-$, $5-H$ or $\left.3^{\prime}-, 5^{\prime}-H\right), 11.09(1 \mathrm{H}$, brs, $\mathrm{NH}) \mathrm{ppm}$; $\delta_{C}\left(100 \mathrm{MHz}, \mathrm{DMSO}-\mathrm{d}_{6}\right) 21.9\left(\mathrm{ArCH}_{3}\right)$, 124.7, 125.2 (3-, 3'-, 5-, 5'-C), 128.3 (3' '-, 5' '-C), 128.9, 131.6 (2-, 2'-, 6-, 6'-C), 130.6 (2',-, 6' '-C), 136.7 (4' '-C), 139.1, 144.7 (1-, 1'-C), 142.9 (1' '-C), 148.7, 149.1 (4-, 4'-C), $150.2(\mathrm{C}=\mathrm{N}) \mathrm{ppm} ; v_{\max }\left(\mathrm{CH}_{2} \mathrm{Cl}_{2}\right.$ thin film) 3197, 1599, 1520, 1406, 1347, 1166, $858 \mathrm{~cm}^{-1}$; $m / z\left(\mathrm{ES}^{-}\right) 439\left([\mathrm{M}-\mathrm{H}]^{-}, 100 \%\right)$.

\section{Bis(4-nitrophenyl) diazomethane}

A suspension of 4,4'-Dinitrobenzophenone (4' '-toluene sulphonyl) hydrazone (0.50 g, $1.1 \mathrm{mmol})$ in $1 \mathrm{M}$ aqueous sodium hydroxide $\left(20 \mathrm{ml}\right.$ ) was heated to $70^{\circ} \mathrm{C}$ for $3 \mathrm{~h}$ then cooled to room temperature. The mixture was extracted with DCM and the combined organics washed with brine and dries over sodium sulphate. Concentration in vacuo yielded the product $(0.32 \mathrm{~g}, 87 \%)$ as a dark orange solid which was used without further purification; $\delta_{H}\left(200 \mathrm{MHz}, \mathrm{CDCl}_{3}\right) 7.45\left(4 \mathrm{H}, \mathrm{d}, J=9.0 \mathrm{~Hz}, 2-, 2^{\prime}-, 6^{-}, 6\right.$ ' $\left.-H\right), 8.30(4 \mathrm{H}, \mathrm{d}$, $J=9.0 \mathrm{~Hz}, 3-, 3^{\prime}-$, 5-, 5'-H) ppm; $\delta_{C}\left(100 \mathrm{MHz}^{\prime} \mathrm{CDCl}_{3}\right) 124.8,124.9$ (2-, 2'-, 3-, 3'-, 5-, 5', 6-, 6'-C), 136.0 (1-, 1'-C), 145.6 (4-, 4'-C) ppm; $v_{\max }\left(\mathrm{CH}_{2} \mathrm{Cl}_{2}\right.$ thin film) 2048, 1584, 1504, 1324, $848 \mathrm{~cm}^{-1} ; \mathrm{m} / \mathrm{z}$ (TOF $\left.\mathrm{EI}^{+}\right) 284\left(\mathrm{M}^{+}, 100 \%\right), 272(45 \%)$; found (TOF $\left.\mathrm{EI}^{+}\right)$ 284.0548, $\mathrm{C}_{13} \mathrm{H}_{8} \mathrm{~N}_{4} \mathrm{O}_{4}$ requires 284.0456.

\section{Synthesis of aminophenyl modified diamond}

A sample of nitrophenyl modified diamond was heated in a $1 \mathrm{M}$ aqueous solution of sodium sulphide nonahydrate for $24 \mathrm{~h}$. The sample was cooled and washed with water to furnish the aminophenyl modified diamond .

\section{Synthesis of fluorescein modified diamond}

A sample of aminophenyl modified diamond was immersed in a $10 \%$ solution of fluorescein isothiocyanate in DCM for $18 \mathrm{~h}$. The diamond sample was then removed then Soxhlet extracted for $24 \mathrm{~h}$. with DCM, water and acetone sequentially before being dried in vacuo to give fluorescein modified diamond

\section{References}

1 W. B. Austin, N. Bilow, W. J. Kelleghan, and K. S. Y. Lau, Journal of Organic Chemistry, 1981, 46, 2280.

2 A. M. S. Nastukov, V. V., Journal fuer Praktische Chemie (Leipzig), 1928, 119, 303.

3 W. G. H. Dauben, Claude F.; Muhs, Merrill A, Journal of the American Chemical Society, 1952, 74, 2082.

4 K. Kikukawa, T. Idemoto, A. Katayama, K. Kono, F. Wada, and T. Matsuda, Journal of the Chemical Society-Perkin Transactions 1, 1987, 1511. 
G. Just, Z. Y. Wang, and L. Chan, Journal of Organic Chemistry, 1988, 53, 1030. 